\title{
EMERGENCY MANAGEMENT AND DECISION SUPPORT SYSTEMS
}

\author{
Prof. Dr. Mohamed M. El Hadi
}

\section{ABSTRACT}

Distributed emergency industrial infrastructure covers the incidents of the factories which have high risks. In general, the high emergencies are those relating to the complex areas and they are unstructured officially, as in the cases with ports, airports, railways, petroleum pipelines, etc. the appropriate human organizations contribute as implementer and participator in emergency management specially the multi-events emergencies as related to the previously prepared plans that need to be changed or realized under the unexpected situations. Therefore, this work highlights the characteristics of both emergency management and that of decision as related to industrial emergency management. It identifies a decision making model and its requirement>s definitions of reasoning mechanism and compare both the passive and active DSS. Intelligent DSS and Intelligent Agent Technology (IAT) and their development in an organization are being discussed in this paper.

Keywords: Emergency Management, Decision making, Intelligent Decision Support Systems, Intelligent Agent Technology

\section{1- CHARACTERISTICS OF EMERGENCY MANAGEMENT:}

The main characteristics of emergency managers must have: qualitative weakly structured knowledge about competencies of their own organizations and other potential partners of emergency managing; a strong managerial skills; direct human assistants; an access to different experts to information about emergency and resources state. They need to cooperate with other emergency managers and work under continuous stress. They are not IT managers.

As related to information available, frequently, emergency managers have limited access to information, information is not complete, uncertain, on different levels of details, too much or too dense various information, difficult or time consuming access to specially requested data.

In connection to decision, emergency managers must take their decision under time and resources constraints. Every decision depends on risk evaluation and managers competencies. It is focused on on what to do in emergency domain (not only how to do), who should intervene, and who should serve as an expert. Planned and just activated actions can be not efficient, and can require immediate modifications. Erroneous human decision can be cause of serious and essential losses.

\section{INDUSTRIAL EMERGENCY MANAGE- MENT:}

Industrial emergency is related to the followings:

- State of risk and / or losses generation.

- Which is over the level accepted by local administration?

- Which is caused by an industrial accident?

Managing the industrial emergency means a control of autonomous functional units by task communication in order to achieve an expected goal in the predefined domain.

The risk is a qualitative indicator of the current state of physical objects proportional to the probability of an event which may generate losses, and to the value of maximal losses could be caused to this object by such event. 
* Risk value can be assessed by event specialists or obtained from experts during knowledge acquisition.

* Risk value depends on many attributes of the risk objects and attributes of its environment.

The losses is a qualitative / quantitative indicator of death, injury, destruction in human, economical, cultural and ecological / environmental sense.

The emergency domain is autonomous functional units: fire, etc. which are characterized by competence (type of investment), and access to information sources. The state of the domain which is the goal is that the emergency managers intend to obtain or consider the most important.

The following figure shows the domain of activity of emergency manager:

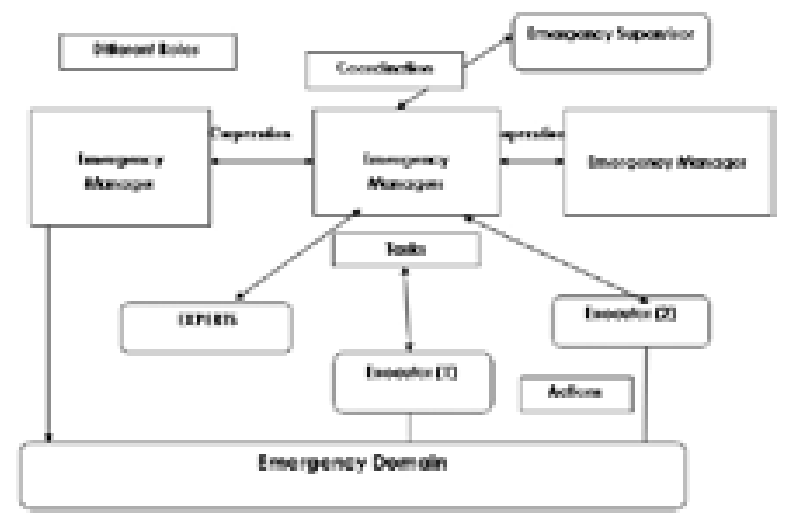

Fig. 1: Domain of Activity of Emergency Manager

\section{DECISION -MAKING MODEL:}

Decision-making model is defined as:

- Decision making $(d-m)$ is a mental activity implied by the necessity of a choice either: without known criteria or without known alternative.

$-\mathrm{K}$

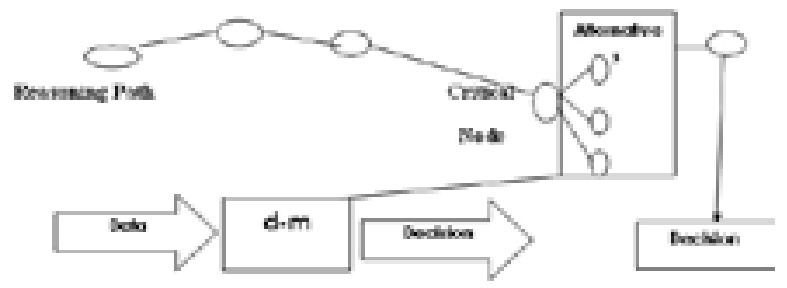

Fig. 2: Decision-Making Model

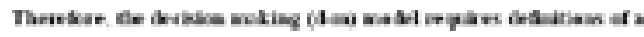

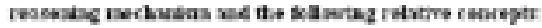

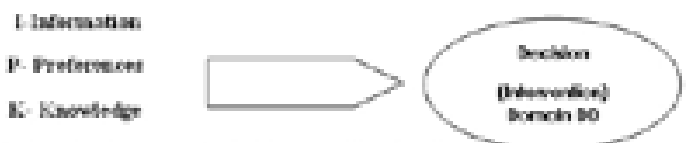

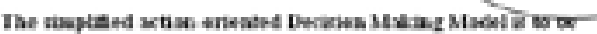
novereed $\mathrm{m}$.

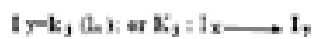

$A,=\mathbf{K},\left(\mathbb{1}_{*}\right) ; \in \mathbb{K},: \mathbf{1}_{\mathrm{s}} \longrightarrow \mathrm{A}$,

I represents states / simulation / changes of the decision domain DD

$\mathrm{K}_{\mathrm{j}}$ represents an inference association on DD

Ko represents an available operation on DD

Ay represents an action on DD

Therefore, simplified D-M Model is as:

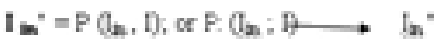

P represents a preference rehtion on DD

1. Sewetes the curredly perferied state ef DD, it can be called intedion max, infedion can be called soal.

I denetes current state on DD

A paeferesce bepeads es the parametet

$\mathrm{P}(\mathrm{X} ; \mathrm{I})$ : if intention is $\mathrm{X}$ and $\mathrm{I} \mathrm{I}_{\mathrm{g}}$

Then intentien is $V$

What is eyulvalent to the setitence:

In the state of DD trean the class lu

$Y$ is better thon $X$

In the reaseeing procetses modeling:

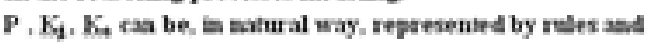
eperabiess (algerithunsi on the level of DD mobel, i.e- they are reforred to chases of informaniva employed in the medel.

In such manner we can construct reasoning paths on the sets of Preferences and Knowledge.

An example of the inference path is shown in the following figure:

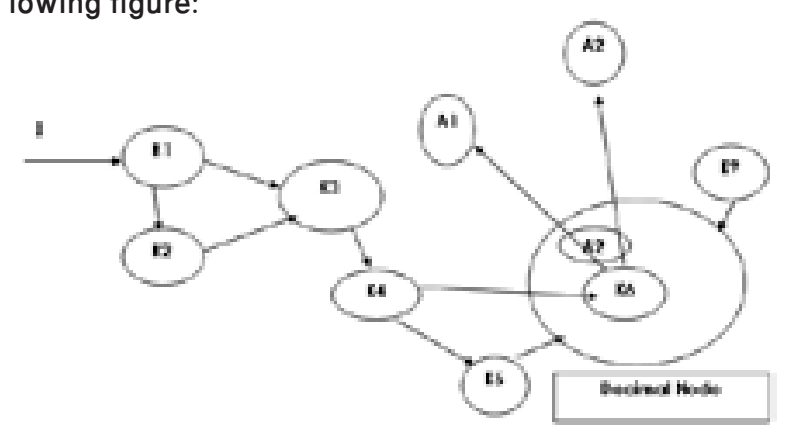

Fig. 3: Interface Path

Here, we may demonstrate that for the decision-making, we need or new information or new preferences or new knowledge. 
Therefore, Decision-Making $(\mathrm{d}-\mathrm{m})$ is a mental activity implied by the necessity of choice either without known criteria or without known alternatives:

- The criteria are to be considered meta-preferences,

- The alternatives are possible actions to be taken.

Therefore Al-

$$
A 2=\mathrm{XS}(1 \mathrm{1})
$$

The fist type of the Dexision-Making rules (meta-preferences)

\section{MP : (ff $A 1_{\Sigma} A X$ and $A 2 \Sigma A Y: \Sigma I M$ then $\left.A 2\right)$}

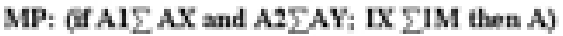

Where AX, AY are classes of actions of the decisionmaker, and IM is a state of Decision Domain (DD).

In such conceptualization, Intelligent Decision support Systems (IDDS) has to have a fixed base of MP rules, such as (in information way);

If is a fire, for example, then activation of fire-men is better than activation of police station for example.

\section{PASSIVE DSS AND ACTIVE DSS (IDSS):}

- Passive classical DSSs provide information,

- Active intelligent DSSs suggest possible actions (knowledge) and inform about used criteria (preferences).

\subsection{Passive DSS:}

Unfortunately the application s of passive DSS requires from their users continuous learning and training to which typical emergency managers are not enough motivated.

Also, a large part of the user decisions relies on the choice of concrete buttom from menu bars or menu tools being parts of a visualized hierarchical menu structures (menu-driven paradigm).

Therefore, Passive DSS gives data and tool choice for decision-making process as identified in the following figure:

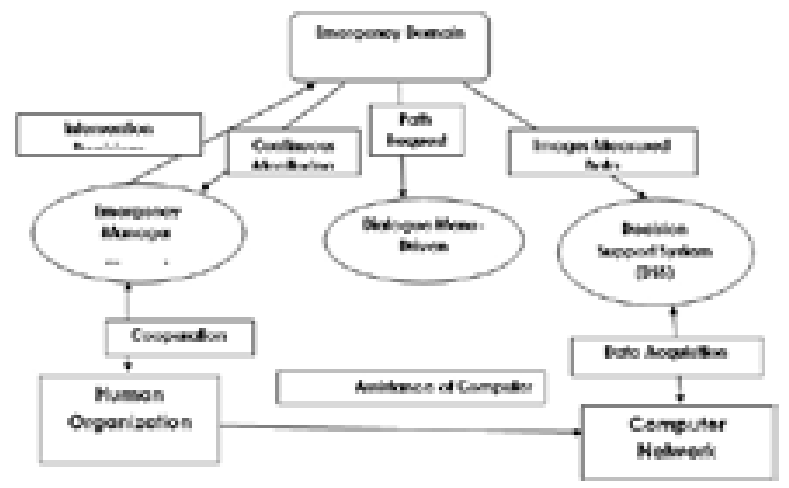

Fig. 4: Passive DSS information System
The passive DSS contains the followings:

- Data Bases, such as Geographic Databases, Dangers Materials DBs, Instructional DB, etc.

- Management Systems,

- Functional Algorithms,

- Emergency Organizational Planners.

\subsection{Why Intelligent DSS?}

IDSSs are especially important when:

- The amount of information necessary for the management is so large, or its time density is so high, that the probability of human errors during emergency decision-making is not negligible.

- The coping with unexpected by managers (DSS designer) situations require from the managers the remembering, mental elaboration and immediate application of complex professional knowledge, which if not properly used, causes fault decisions.

The following figure shows the overview of the IDDS:

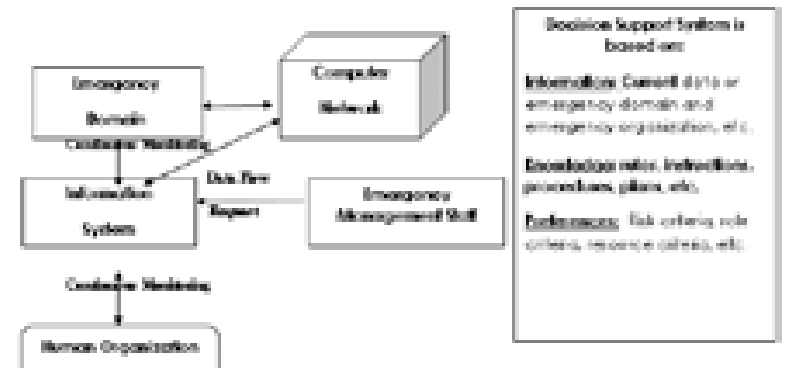

Fig. 5: IDSS Overview

From the above shown figure, IDSS is to include the following suggested:

- Cooperation,

- Experts,

- Intervention, and

- Executors.

Answering the question of how to do the IDSS? It sjould be based on the followings:

- Application of a generic ideal model of decision maker (his role), and

- Its decomposability into human and computer decision-makers.

This is illustrated in the following figure: 


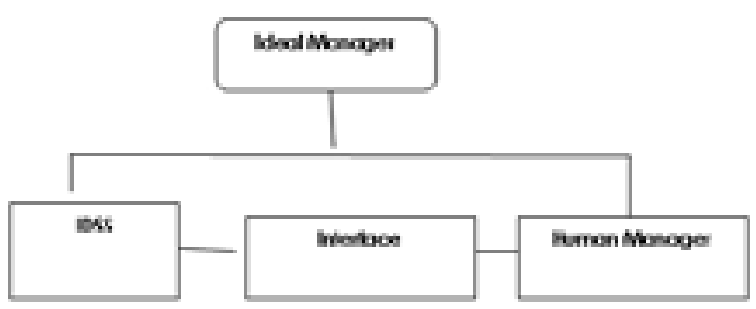

Fig. 6: Modeling the IDSS

\section{INTELLIGENT AGENT TECHNOLOGY (IAT):}

The intelligent agent technology (IAT) offers various tools to suggest classical passive menu driven DSSs to be «intelligent». Specific advantage is autonomy of intelligent agents in task execution. Intelligent agent has capabilities to: Information filtering and interpretation according to the manager role and situation model. It may suggest new goals, alternative decisions or elaborate plans of intervention. Intelligent agent can use various Artificial Intelligence (Al) methods whih enable to copy with uncertain and incomplete data, qualitative reasoning, constraints satisfactions, etc.

The flexibility of IAT, modularity and reusing depend strongly on the type of architecture accepted. An «organization» of task-independent intelligent agent can be considered as the kernel of IDSS. Now, a multi-agent architecture is based on a repetitive structure, the possibility of (user friendly) modifications of the specific emergency domain and user roles, are considered as a key research field in the IDSS development.

The following three figures are related to IDSS/IAT framework, development and types architecture:

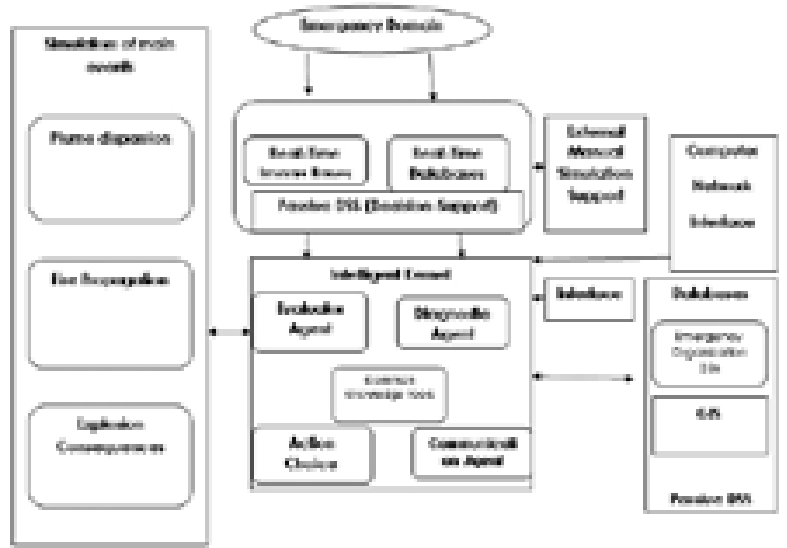

Fig. 7: IDSS/IAT Frame System

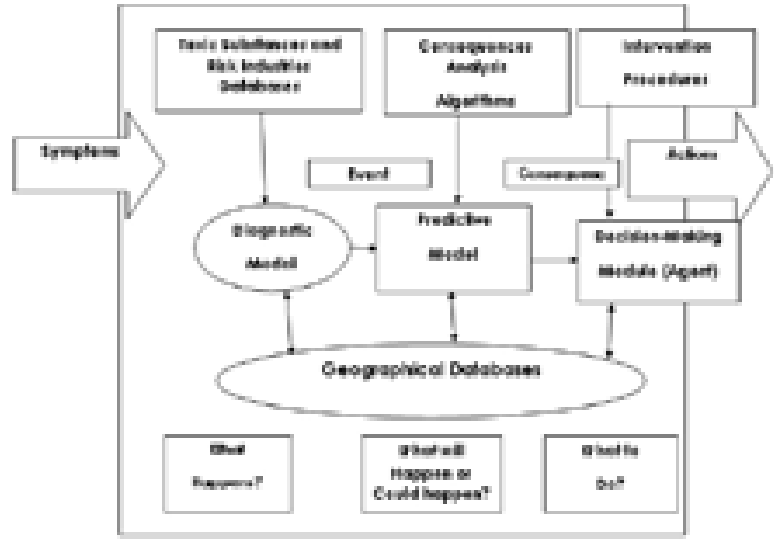

Fig. 8: IDSS/IAT Development

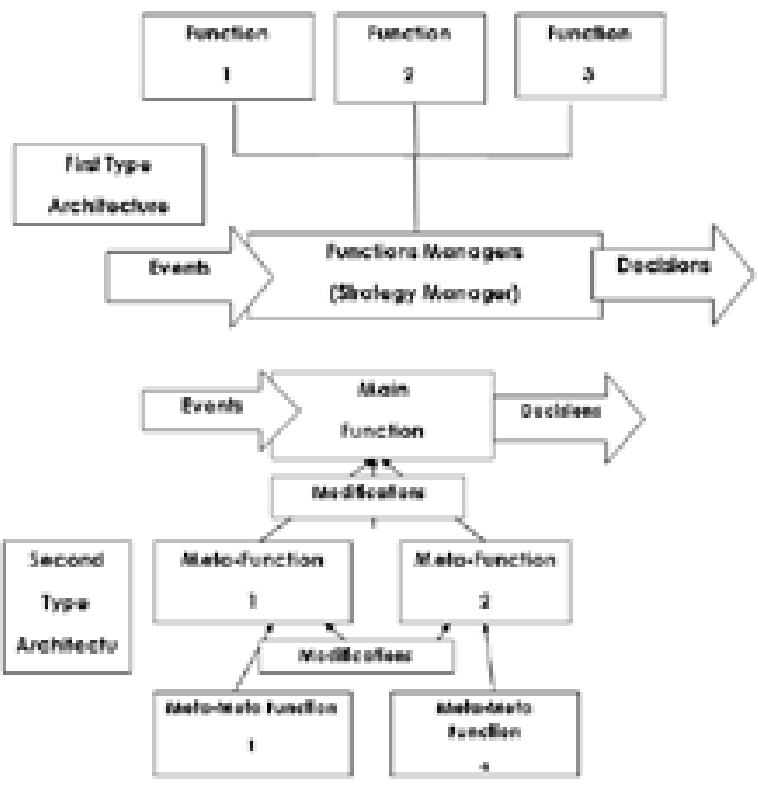

Fig. 9: IDSS/IAT Types Architecture

From the above figures related to the IDSS and IAT frame system, development and types architecture we could have:

*A structural intelligence of multi-agent system, or *Behavioral intelligence of multi-functional system. Therefore the following four figures are related to the structural intelligence whether to the abstract simple agent or the physical domain of activity; a multi-level abstract intelligent agent architecture; a decision-making module based on abstract-intelligent agent architecture; and decision-making module example of a domain-representation module. 

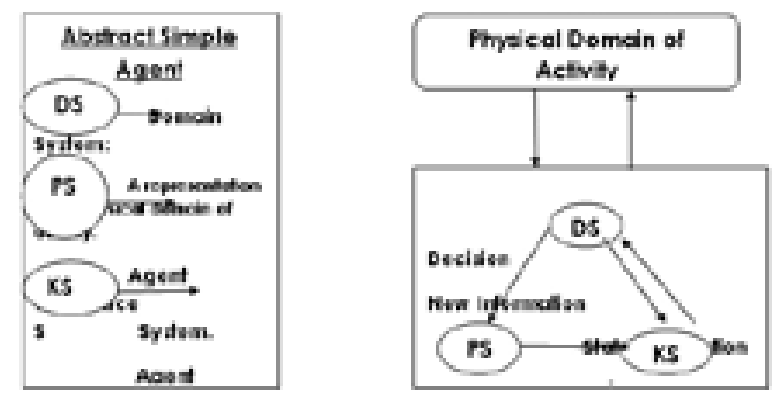

Fig. 10: IDSS/IAT Structural Intelligence

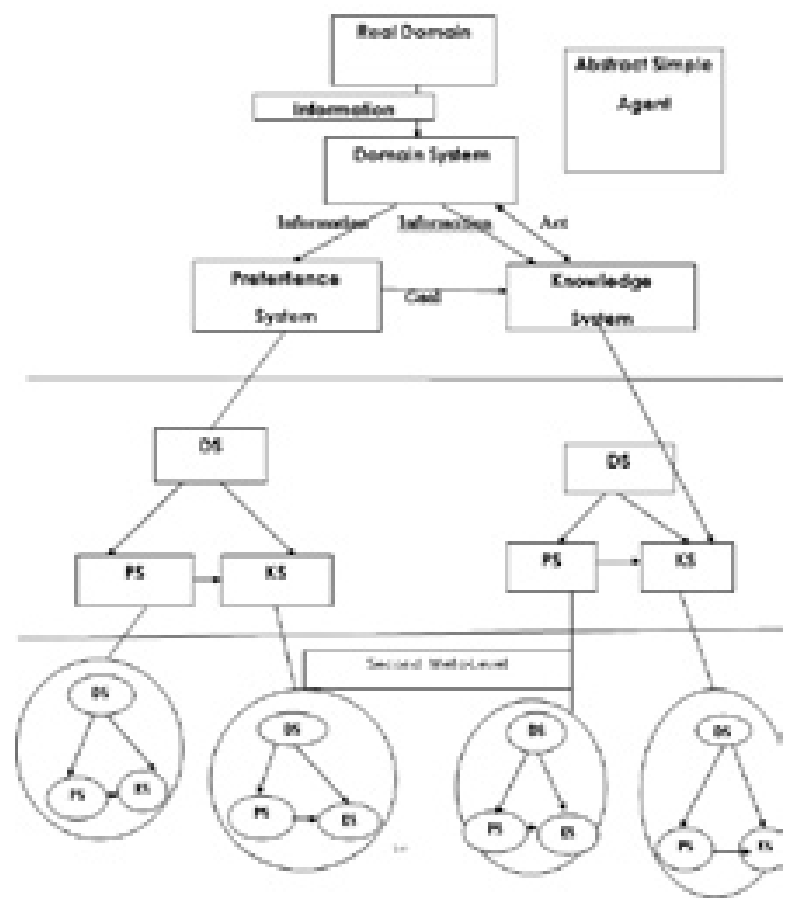

Fig. 11: Multi-Level Abstract Intelligent Agent Architecture
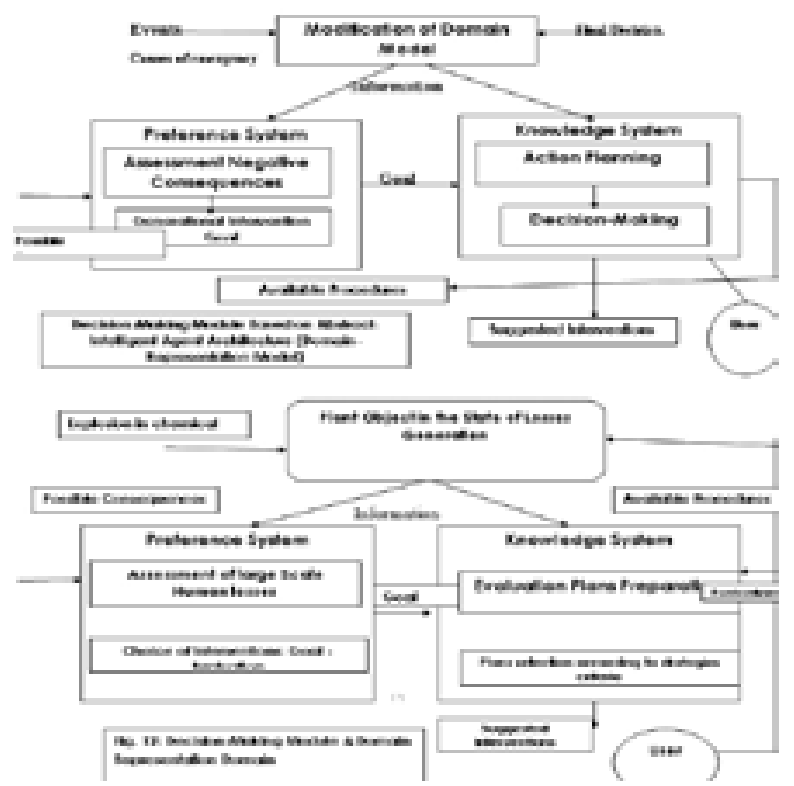

lowings:

1. New ontology as related to DSS problems which are re-conceptualized.

2. Strong interdisciplinary approach.

3. New technologies concerning reasoning tools and intelligent agent architecture.

4. New possibilities of uncertain, complex and high risk domain management

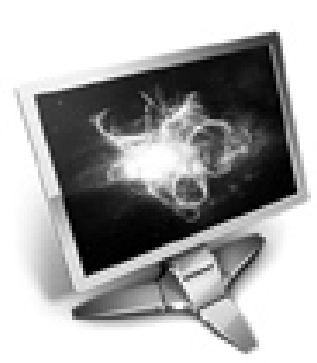

